Copyright by the American Institute of Physics (AIP). Dong, SX; Bai, FM; Yan, L; et al., "Vacuum response and gas leak detection in piezoelectrically driven sound-resonance cavity," Appl. Phys. Lett. 84, 4144 (2004); http:// dx.doi.org/10.1063/1.1751217

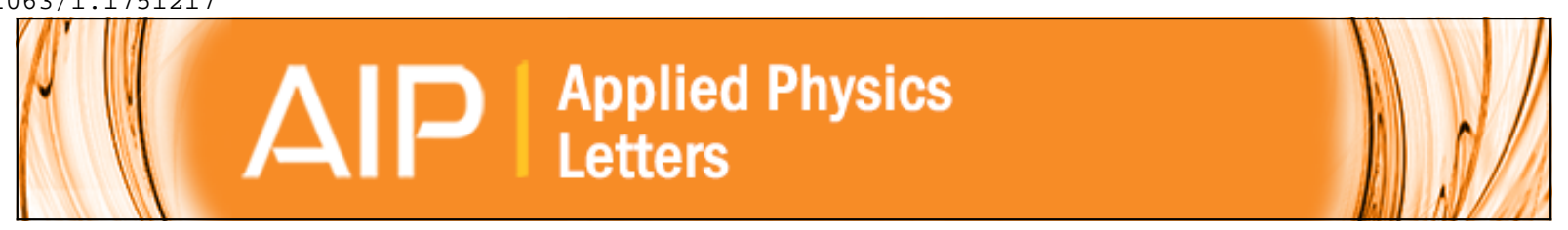

Vacuum response and gas leak detection in piezoelectrically driven sound-resonance cavity

Shuxiang Dong, Feiming Bai, Li Yan, Hu Cao, Jie-Fang Li, and D. Viehland

Citation: Applied Physics Letters 84, 4144 (2004); doi: 10.1063/1.1751217

View online: http://dx.doi.org/10.1063/1.1751217

View Table of Contents: http://scitation.aip.org/content/aip/journal/apl/84/21?ver=pdfcov

Published by the AIP Publishing

Over 700 papers \&

presentations on

multiphysics simulation visw now

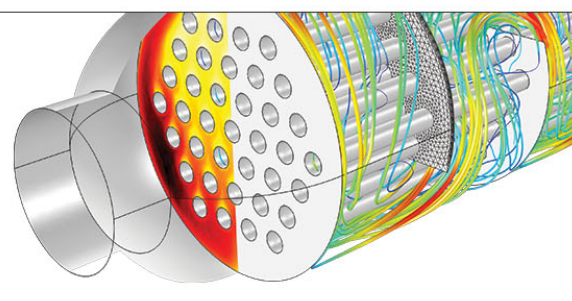




\title{
Vacuum response and gas leak detection in piezoelectrically driven sound-resonance cavity
}

\author{
Shuxiang Dong, ${ }^{\text {a) }}$ Feiming Bai, Li Yan, Hu Cao, Jie-Fang Li, and D. Viehland \\ Materials Science \& Engineering, Virginia Tech, Blacksburg, Virginia 24061
}

(Received 20 January 2004; accepted 22 March 2004; published online 6 May 2004)

\begin{abstract}
It has been found that a piezoelectrically driven sound-resonance cavity (PSRC) is quite sensitive to low-pressure changes: a change from ambient to vacuum $(67 \mathrm{~Pa})$ conditions results in a $89^{\circ}$ phase shift and a $-81 \%$ voltage decrease. Under vacuum conditions, the PSRC is highly sensitive $(\sim 7$ ppm) to leakage of $\mathrm{H}_{2}$ gas. (C) 2004 American Institute of Physics. [DOI: 10.1063/1.1751217]
\end{abstract}

In prior articles, ${ }^{1,2}$ we reported the development of a piezoelectric sound-resonance cavity (PSRC), which exhibited an extremely high sensitivity to $\mathrm{H}_{2}$ gas concentration changes over a broad concentration range. The sensitivity is due to a shift in the sound-resonance state of the cavity with $\mathrm{H}_{2}$ concentration changes. Here, we will further show that the PSRC is also sensitive to vacuum pressure changes, due to gas density variations. The mechanism of vacuum detection by the PSRC is different from that of thermal conductivity, ${ }^{3-7}$ thermal resonance, ${ }^{8}$ rf pulse transmission, ${ }^{9}$ optical Pirani gauges, ${ }^{10}$ electrostatic capacitors, ${ }^{11}$ and quartz resonators. ${ }^{12}$

A PSRC consists of a small cylindrical-type cavity and two thin disk-type piezoelectric bimorphs. A piezoelectric disk is attached to each end of the cavity, where one of the disks has a center hole. One of the piezoelectric disks is used to produce a bending vibration under a small applied ac voltage $\left(V_{\text {ac }}\right)$, forcing the gas in the cavity to oscillate and diffuse. At resonance, the gas will be in a standing wave sound resonance state. The second piezoelectric element at the opposite end of the cavity acts as a sensing element, capable of monitoring changes in both the acoustic intensity $(I)$ and the phase $(P)$ of a standing wave. As a result, the piezoelectric sensor will produce voltage $\left(V_{s}\right)$ and phase $\left(P_{s}\right)$ signals which are proportional to $I$ and $P$, respectively.

The resonance frequency of a cavity containing air of a nominal composition (assuming ideal gas behavior) is

$$
f_{0}=K C_{\text {air }}=K \sqrt{\frac{\gamma P}{\rho}}
$$

where $K$ is a geometric parameter related to the structure and size of the cavity, $C_{\text {air }}$ is the sound velocity of the gas, $\gamma$ is the adiabatic constant (1.4 for air), $P$ is the air pressure and $\rho$ is the gas density. When air pressure decreases, both $\rho$ and $P$ will decrease simultaneously; as a result, $C_{\text {air }}$ is nearly unchanged with decreasing pressure. ${ }^{13}$ However, the change in $\rho$ will also result in a change in the characteristic acoustic impedance of the gas (i.e., $\rho C_{\text {air }}$ ); and since $C_{\text {air }}$ is constant, we have

$$
\Delta\left(\rho C_{\text {air }}\right) \sim C_{\text {air }} \Delta \rho .
$$

\footnotetext{
a) Author to whom correspondence should be addressed; electronic mail: sdong@vt.edu
}

If the piezoelectric driving element is then operated under a constant vibration velocity, the gas particles will diffuse in the cavity at a constant average speed $v_{e}$. As a result, any change in the acoustic impedance will cause a change in the acoustic intensity radiation $(\Delta I)$, given as

$$
\Delta I=C_{\text {air }} v_{e}^{2} \Delta \rho .
$$

According to Eq. (3), if $v_{e}$ is constant, a decrease in the gas density will result in a lower acoustic intensity radiation. Because the voltage signal $V_{s}$ induced on the sensing element is proportional to $\Delta I$, a small change in air density will result in a proportional change in $V_{s}$. Accordingly, there is a direct linear relationship between the acoustic properties of the cavity and the gas density there within, which can be measured by the voltage output from a piezoelectric sensing element. In addition, a gas density change in the cavity will cause a phase shift in the sound-resonance standing wave. A sound-resonance standing wave is a result of superposition of the incident wave (e.g., produced by the piezoelectric driver) and the reflected wave (e.g., from the piezoelectric sensing element), and thus any change in acoustic impedance of the cavity will result in a phase shift of the superposition wave. ${ }^{14}$

Two PSRC gas sensors with operational frequencies of 4.3 and $4.8 \mathrm{kHz}$ were constructed. ${ }^{1}$ Prototype 1 consisted of two disk-type PZT thin layers with a diameter of $8 \mathrm{~mm}$ and a thickness of $100 \mu \mathrm{m}$, and a plastic cavity of $7 \mathrm{~mm}$ in diameter and of $3 \mathrm{~mm}$ in height. Its resonance frequency was $\sim 4.3 \mathrm{kHz}$. Prototype 2 also consisted of two PZT disks, but had a slightly smaller cavity. Its resonance frequency was thus a little higher $(\sim 4.8 \mathrm{kHz})$. The PSRC sensors were operated in a $90^{\circ}$ (or $-90^{\circ}$ ) phase state under constant frequency conditions, as these states exhibited the highest sensitivity to gas density variations. During operation, the resonance standing wave of the PSRC will cause its cavity volume to expand/contract cyclically (over 4000 times/s). During expansion the cavity will draw gas into itself, whereas during contraction it will force gas out. This will result in an acoustic impedance change, which can be detected as a voltage signal $\Delta V_{s}$ and as a phase shift $\Delta P_{s}$. The rapid nature of the gas exchange will result in a fast response time to density fluctuations within the cavity.

Measurements of the two PSRC sensors were performed in a vacuum test chamber. A small ac $(4.3-4.8 \mathrm{kHz})$ voltage signal of $0.2 \mathrm{~V}$ (rms) was used to drive a piezoelectric ele- 


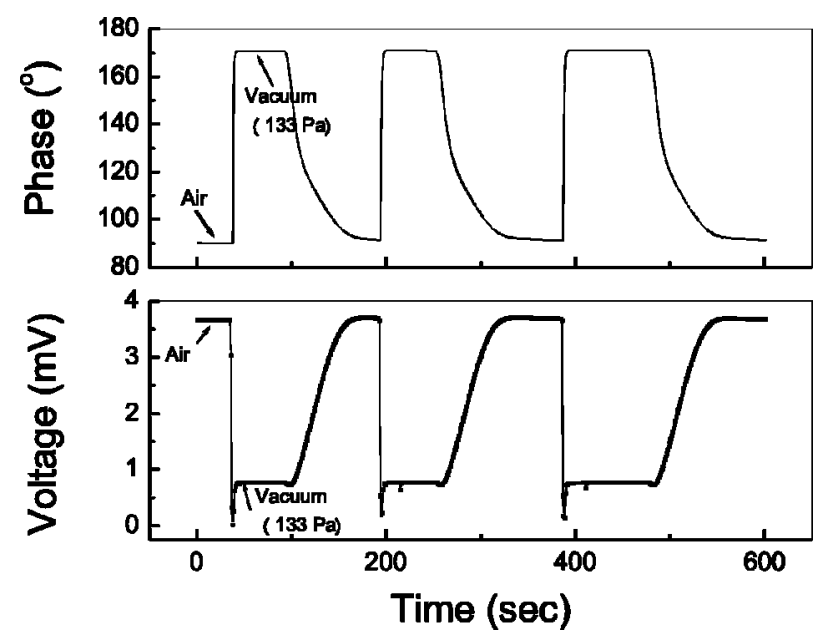

FIG. 1. Phase shift $P_{s}$ and voltage change $V_{s}$ of PSRC prototype 1 as a function of time in response to a change in air pressure from ambient to low pressure (133 Pa). The driving frequency of this PSRC was $4.30 \mathrm{kHz}$, and the drive voltage was $0.2 \mathrm{~V}_{\mathrm{rms}}$.

ment at one end of the cavity. A lock-in amplifier was used to monitor the signal from the piezoelectric sensing element at the other end. The temperature and relative humidity were held constant in the chamber. To characterize the vacuum response of the PSRC, the gas pressure in the chamber was varied over a wide range from ambient conditions (1.01 $\left.\times 10^{5} \mathrm{~Pa}\right)$ to a low pressure of $66.7 \mathrm{~Pa}$.

Figure 1 shows the phase (part a) and voltage (part b) responses of our PSRC prototype 1 as a function of time after several successive changes in air pressure from ambient to low pressure $(133 \mathrm{~Pa})$. On going from ambient to vacuum conditions, it can be seen that both responses are rapid, occurring in a stepwise manner; that the phase shift is $\sim 80^{\circ}$ (89\%); and that the voltage change is $-3.0 \mathrm{mV}(81 \%)$. Clearly, when the gas density decreases, the voltage signal from the piezoelectric sensing element is also decreased, as predicted by Eq. (3).

In the low air pressure range from 101 to $30 \mathrm{kPa}$, the PSRC exhibited a near-linear response to air pressure changes. Figure 2 shows the output voltage and phase angle of the PSRC prototype 2 as a function of air pressure in this
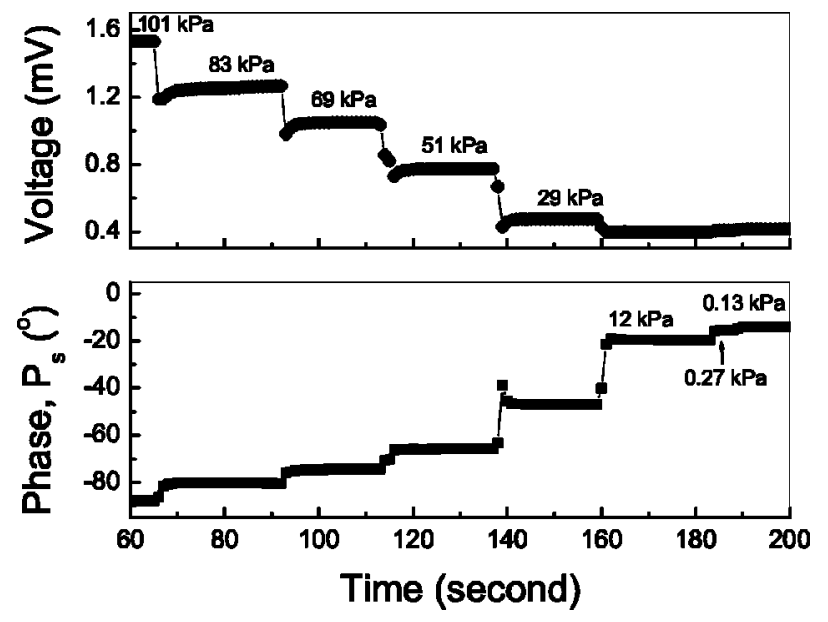

FIG. 2. Phase $P_{s}$ and voltage $V_{s}$ of PSRC prototype 2 as a function of low air pressure. The driving frequency of this PSRC was $4.79 \mathrm{kHz}$, and the drive voltage was $0.2 \mathrm{~V}_{\mathrm{rms}}$.
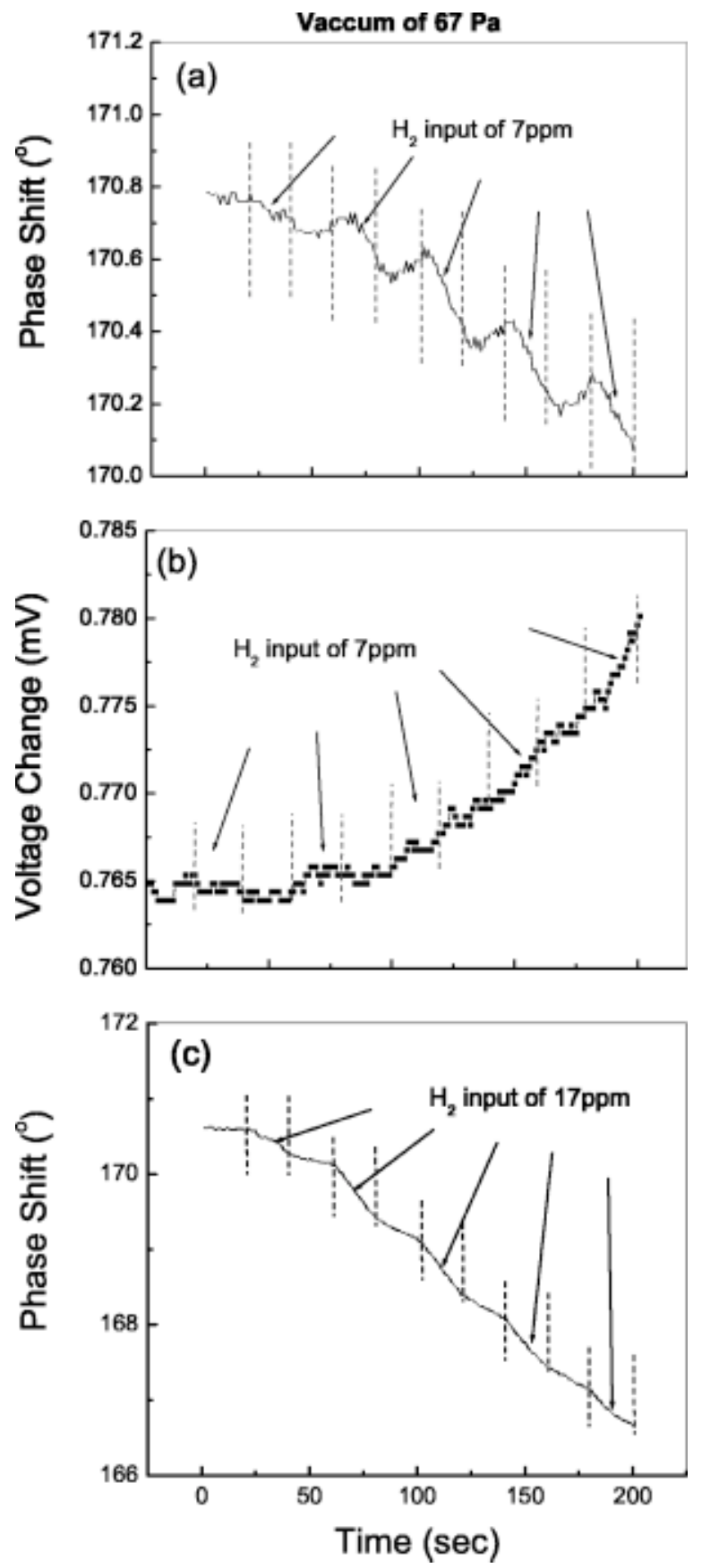

FIG. 3. PSRC sensor (prototype 1) response as a function of time to a $\mathrm{H}_{2}$ gas input of $7 \mathrm{ppm}$ every $20 \mathrm{~s}$ under a low-pressure condition of $67 \mathrm{~Pa}$ : (a) phase $P_{s}$ and (b) voltage $V_{s}$; and (c) phase shift $P_{s}$ in response to $\mathrm{H}_{2}$ gas inputs of $17 \mathrm{ppm}$ every $20 \mathrm{~s}$ under a vacuum condition of $67 \mathrm{~Pa}$. Constant temperature $\left(25^{\circ} \mathrm{C}\right)$ and humidity $(43 \%)$ were used for all measurements.

range. However, when the air pressure was decreased to $<0.1 \mathrm{kPa}$ (i.e., close to vacuum), the change in both the phase and voltage signals tended to saturate, presumably due to lack of gas medium.

Under vacuum conditions, the PSRC exhibited a high sensitivity to insertion of minute quantities of $\mathrm{H}_{2}$, leaked into the cavity. $\mathrm{H}_{2}$ gas has much different sound acoustical properties than air of normal ambient composition. ${ }^{2}$ Consequently, leakage of $\mathrm{H}_{2}$ into the cavity will result in a shift of the sound-resonance state of the PSRC prototype 2, and thus a voltage increment $\left(\Delta V_{s}>0\right)$ and phase shift $\left(\Delta P_{s}<0\right)$ will be detected by the piezoelectric sensing element. Figure 3 shows the response of the PSRC prototype 1 to input $\mathrm{H}_{2}$ 
gas concentration changes in aliquots of $\sim 7$ and $17 \mathrm{ppm}$ every $20 \mathrm{~s}$, beginning from a low pressure of $67 \mathrm{~Pa}$. It can be seen in parts (a) and (b) of this figure that a leakage of $7 \mathrm{ppm}$ results in a phase shift of about $-0.1^{\circ}$ and a voltage change of $\sim 5 \mu \mathrm{V}$, respectively. The phase shift was found to be more sensitive at low vacuum conditions than at higher pressures. It is also important to note that the response time of the PSRC to $\mathrm{H}_{2}$ gas leakage was quite rapid $<2 \mathrm{~s}$, offering in situ sensing capabilities in real time.

Due to its unique acoustical sensing mechanism and fast response time, we believe that the PSRC sensors may be quite useful for leak detection of many types of gases. Under normal operation, the phase and voltage from the piezoelectric elements of the sensor will be constant; however, if leakage occurs, then both a phase shift and a voltage change will be detectable. In particular, because $\mathrm{H}_{2}$ has a much lower molecular weight than other gaseous species, the PSRC could find use as a miniature $\mathrm{H}_{2}$ leak detector-especially prospective are applications where $\mathrm{H}_{2}$ is used as a fuel under low-pressure conditions. It is also worth noting that the PSRC offers an ease of integration with composite structures (e.g., fuel tanks and lines), as it is small, robust, lightweight and low power consuming.

In summary, it has been shown that a piezoelectrically driven sound-resonance cavity can be used as a gas leak detector under low-pressure conditions. We have prototyped two PSRC sensors, analyzed their low-pressure and vacuum responses, and determined the operational mechanism of the PSRC. Investigations have demonstrated high sensitivity to minute $\mathrm{H}_{2}$ gas concentration changes under low-vacuum conditions, within a rapid response time of $t<2 \mathrm{~s}$.

This research was supported by the NASA, Marshall Space Flight Center. Any opinions, findings, and conclusions or recommendations expressed in this material are those of the author(s) and do not necessarily reflect the views of NASA.

${ }^{1}$ S. Dong, F. Bai, J. F. Li, and D. Viehland, Appl. Phys. Lett. 82, 4590 (2003)

${ }^{2}$ S. Dong, F. Bai, J. F. Li, and D. Viehland, IEEE Trans. Ultrason. Ferroelectr. Freq. Control 50, 1105 (2003).

${ }^{3}$ S. A. Adamovsky, A. A. Minakov, and C. Schick, Thermochim. Acta 403, 55 (2003).

${ }^{4}$ T. M. Berlicki, Sens. Actuators, A 93, 27 (2001).

${ }^{5}$ T. M. Berlicki, J. Vac. Sci. Technol. A 19, 325 (2001).

${ }^{6}$ G. Bedo, W. Kraus, and R. Muller, Sens. Actuators, A 85, 181 (2000).

${ }^{7}$ T. M. Berlicki, Vacuum 57, 413 (2000).

${ }^{8}$ B. Folkmer, A. Siber, and W. G. Bley, Sens. Actuators, A 74, 90 (1999).

${ }^{9} \mathrm{H}$. Li and A. Lal, Boston Transducers' 03, Digest of Technical Papers 1, 53 (2003).

${ }^{10}$ K. B. Brown, Y. Ma, R. P. W. Lawson, W. Allegretto, F. E. Vermeulen, and A. M. Robinson, Can. J. Electr. Comput. Eng. 27, 27 (2002).

${ }^{11}$ H. Miyashita and M. Esashi, J. Vac. Sci. Technol. B 18, 2692 (2000).

${ }^{12}$ P. C. Chen and L. Wu, Jpn. J. Appl. Phys., Part 1 39, 2710 (2000).

${ }^{13}$ D. E. Caro and L. H. Martin, Proc. Phys. Soc. B 66, 760 (1953).

${ }^{14}$ P. M. Morse and K. Uno. Ingard, Theoretical Acoustics (McGraw-Hill, New York, 1968). 